\title{
Propagation of Radial Pulsation Modes in the Outer Atmosphere of Arcturus: First Results
}

\author{
M. Cuntz \\ High Altitude Observatory, National Center for Atmospheric Research ${ }^{1}$, \\ Boulder, CO 80307-3000, USA
}

\begin{abstract}
I present first results of a study about propagating radial pulsation modes in the outer atmosphere of Arcturus (K1.5 III). Mechanical energy input is explicitly taken into account by treating shock wave dissipation. I investigate the influence of different wave frequencies on the mass loss behavior of the star. I show that significant time-averaged mass loss can only be produced when periods larger than $5 \times 10^{5} \mathrm{~s}(\sim 1$ week) are employed. The initial atmosphere I use extends from $1.2 \mathrm{R}_{*}$ up to $11.8 \mathrm{R}_{*}$. All wave models are adiabatic. I found that the mass loss rates and the final flow speeds obtained are extremely sensitive to the wave periods. In a certain regime the effect of increasing the period by a factor of 4 is to increase the corresponding mass loss rate by four orders of magnitude. I note that the mass loss rate and final flow speed of the wind for a $5.6 \times 10^{5} \mathrm{~s}$ period wave are somewhat close to the observed values. A more complete discussion regarding mass loss generation in Arcturus has been presented by Cuntz (1990). Recent observations of low-amplitude radial velocity variations in the photosphere of Arcturus provide evidence that the theoretically predicted mass loss frequencies might exist. Belmonte et al. (1990) presented evidence for a $\sim 8.3 \mathrm{~d}$ period with an amplitude of $\sim 50 \mathrm{~m}$ $\mathrm{s}^{-1}$, which they attributed to the fundamental radial pulsation mode. Further studies are in progress (Larson et al. 1992). I note that if the $8.3 \mathrm{~d}$ period is real than it would be sufficiently large to support continuous mass loss. Judge \& Stencel (1991) argued that the mechanical energy in the observed disturbances could be $\sim 15$ times greater than the energy required to drive the wind. For other stars than Arcturus the required minimum mass loss periods can be estimated as $\mathrm{P}_{M L} \sim \mathrm{R}_{*}$.
\end{abstract}

\section{References:}

Belmonte J.A., Jones A.R., Pallé P.L., \& Cortés T.R.: 1990, ApJ, 358, 595

Cuntz M.: 1990, ApJ, 353, 255

Judge P.G., \& Stencel R.E.: 1991, ApJ, 371, 357

Larson A., Irwin A.W., Yang S., Goodenough C., Walker G.A.H., Bohlender D.A., \& Walker A.R.: 1992, this meeting

1 The National Center for Atmospheric Research is sponsored by the National Science Foundation 